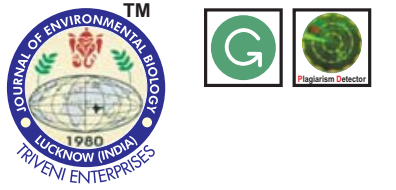

\title{
Identification of metabolomic changes before and after exercise regimen in stress induced rats
}

\section{Authors Info \\ R. Sowndarya and V.A. Doss* \\ Department of Biochemistry, \\ PSG College of Arts \& Science, \\ Coimbatore-641 014, India}

${ }^{*}$ Corresponding Author Email : victordoss64@gmail.com

Key words

Chronic Unpredictable Mild Stress,

Forced swim test,

Metabolomic changes,

Serum level

Publication Info

Paper received : 09.09.2016

Revised received : 19.01.2017

Re-revised received : 20.02 .2017

Accepted: 08.03.2017

\section{Abstract}

Aim : To identify the metabolomic changes before and after exercise regimen in depression induced animal models.

Methodology : Severity of depression was measured by forced swim test (FST) and sucrose consumption test (SCT) and their statistical significance was obtained by ANOVA followed by post hoc test. Swimming protocol was followed for 4 weeks of exercise treatment. Serum obtained from depressed and exercise treated rats were used for the metabolite analysis by GCMS. Subsequent statistical analysis (ANOVA followed by post hoc test) revealed significant metabolic changes.

Results : About 20 metabolites were found to be differentially expressed in control, depressed and exercise treated groups. Serum levels of glycine and serine were significantly increased in depressed groups, whereas the levels of leucine, proline, valine (BCAs), glucose, fructose, galactose, mannose, xylitol myo-inositol, lactic acid, oleic acid, palmitic acid, stearic acid were significantly decreased when compared to control group (Group I). After 4 weeks of swimming exercise regimen procedure, lower levels of glycine, serine and higher levels of sugars, myo-inositol, oleic acid, palmitic acid, stearic acid and BCAs were found in the exercised groups (Group III) when compared to the depressed groups (Group II).

Interpretation : These observations suggested that the depressed state may be associated with the changes in the level of few metabolites involved in amino acid, fatty acid, glucose and energy metabolisms, which may get reverted after chronic exercise.

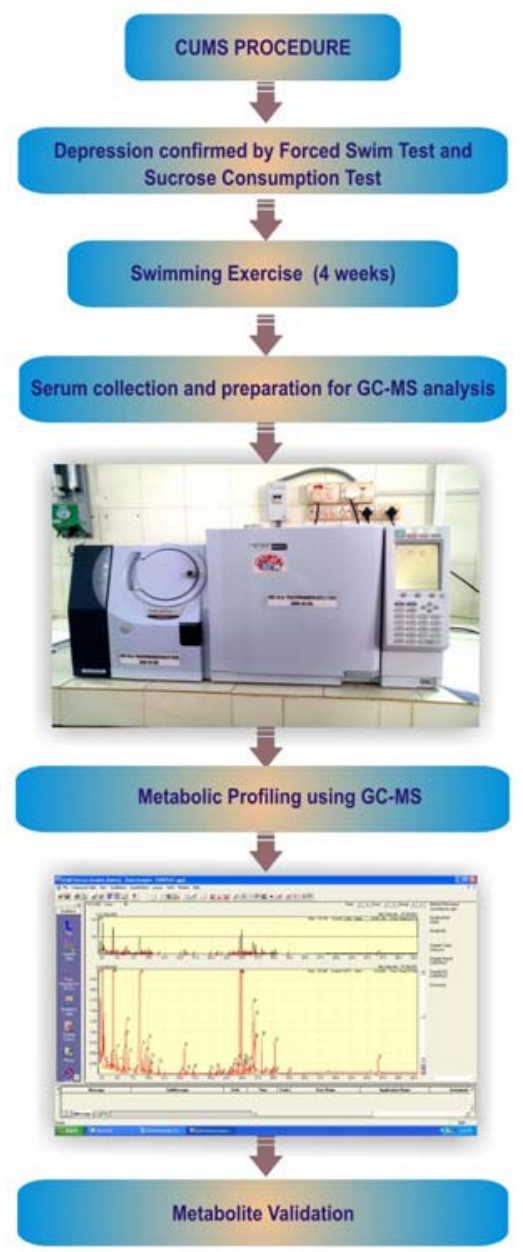




\section{Introduction}

Depression is a serious psychiatric disorder often associated with stressful events that can lead to emotional and physical problems (Shenl et al., 2003). The global burden percentage of depression is about $12.3 \%$ and is predicted to rise by $15 \%$ in 2020 (Reynolds, 2003). Cognitive and emotional biases play an important role in the development of depression (Anisman and Matheson, 2005; Coles and Heimberg, 2002). Chronic unpredictable mild stress (CUMS) is a well-validated animal model that mimics several human symptoms of depression (Willner, 1997) and had been used widely for studying clinical depression and effect diverse antidepressant drugs (Redrobe et al., 1998).

Metabolomics enables the investigation of the metabolic response of the living system by measuring the variations in the metabolic profiles in biological fluids (Kell, 2006). Metabolomics is a non-targeted approach used in the study of metabolic networks for identifying disease-specific metabolic profiles and biomarkers of CNS disorders (Schwarz and Bahn, 2008; Kadddurah - Daouk et al., 2008). Gas chromatography - mass spectrometry (GC-MS) is one of the most efficient, sensitive and reliable metabololite detection tool due to its high resolution and selectivity. It produces reproducible molecular fragmentation patterns making it an integral tool for metabolite identification (Pasikanti et al., 2008).

Metabolomics was used to study the pathogenesis of major depressive disorder (Wingenfeld and Wolf, 2011). There is a promising evidence that exercise can increase synaptic plasticity, enhance neuronal survival, while the stress shows contradictory effects, which indicates a potential mechanism for exercise to reduce stress (Cotman et al., 2007). A previous study has reported the antidepressant like effect of exercise which promotes neurogenesis and inhibits neurodegeneration (Eyre and Baune, 2012).

In the present study, Chronic Unpredictable Mild Stress was induced to produce gradual development of depression. The central objective of this study was to investigate the metabolic changes in the serum of control, depressed and exercised rats. It was hypothesized that depressed rats, subjected to moderate swimming exercise for 28 days would show up some changes in their metabolic profile that may bring out some reduction in the stress level. To our knowledge, this is the first analysis comparing metabolomic changes in the serum of depressed and exercised animal models.

\section{Materials and Methods}

Animals : Female Wistar rats weighing about 150 - 200g were purchased from Kovai Medical Center and Hospital, Tamilnadu, India. Animals were maintained at constant temperature $\left(37^{\circ} \mathrm{C}\right)$, humidity ( $45 \pm 15 \%$ ) controlled room with $12 \mathrm{hrs}$ light/dark cycle and free access to food and water. The ethical clearance for handling of experimental animals was obtained from the
Institutional Animal Ethics Committee (IAEC) of PSG Institute of Medical Sciences and Research.

Chronic Unpredictable Mild Stress (CUMS) procedure : Rats were randomly divided into three groups ( $n=6$ per group): control group, depression-induced group and exercise treated group. The control rats were housed together without any disturbance and the model rats were exposed to the following CUMS procedure following the method of Willner, (1997) : cage tilting (tilt at $45^{\circ} \mathrm{C}$ angle) for $24 \mathrm{hrs}$, crowded housing (5 rats per cage), noises for $1 \mathrm{hr}$ (alternative periods of noise $10 \mathrm{~min}$ and silence 10 min), 24 hrs food, 24 hrs water deprivation, soiled bedding (wetting cage with water), removal of bedding materials for $12 \mathrm{hrs}$, reversed light-dark cycle respectively. One stressor was applied per day and the whole stress process was done in a random order for 4 weeks and at the end of it, depressive behaviour was confirmed by forced swim test and sucrose consumption test.

\section{Behaviour test}

Forced swim test : The forced swim test was carried out according to Porsolt et al. (1977). Here, the experimental rats were made to swim individually in a cylindrical container of $35 \mathrm{~cm}$ in height and $15 \mathrm{~cm}$ in diameter, such that the rats could not touch the bottom of the cylinder with its limb or tail or climb over the edge of the chamber.

Trial sessions were conducted before the start of actual 6 min test. The initial 2 min in the total 6 min were not considered, because during that time period, animals treid to escape. A rat was considered immobile when it remained floating in the water without struggling, making only minimum movements to keep its head above water. The total duration of immobility during final 4 min were recorded. The rats were then allowed to dry in a prewashed enclosure $\left(\sim 32^{\circ} \mathrm{C}\right)$ before being returned to their cages.

Sucrose consumption test : This test was performed following the method of to Willner et al. (1987). After $24 \mathrm{hr}$ period of food and water deprivation, each rat was rested to be in individual cage and was given two bottles containing $1 \%$ sucrose solution and water. The preference for sucrose was calculated as a percentage of sucrose solution consumed in the total amount of liquid intake.

Exercise protocol : Rats were trained in a moderate swimming program according to the method of Liu et al. (2010). Daily swimming exercise was performed in a large plastic barrels (45 $\mathrm{cm}$ in diameter and $60 \mathrm{~cm}$ in height) filled with fresh water $\left(32^{\circ} \mathrm{C} \pm\right.$ $2^{\circ} \mathrm{C}$ ) to the depth of $50 \mathrm{~cm}$. Exercise was performed at the same time daily (between 10.00 a.m. to 12.00 p.m.). After swimming, the rats were towel dried and kept warm by electric drier.

The swimming program included two phases: adaptation and training. During first week (adaptation), the training begun for $15 \mathrm{~min}$ on the first day and it was gradually increased for 60 min on the last day. The adaptation was performed for reducing the water induced stress without promoting physiological alterations in 
relation to physical training (Contarteze et al., 2008).

Analysis of metabolites : Rats were sacrificed and the blood collected was centrifuged at $4000 \mathrm{rpm}$ for $20 \mathrm{~min}$ at $4^{\circ} \mathrm{C}$ to separate the serum. The serum was stored at $-20^{\circ} \mathrm{C}$ and used for the analysis of amino acids, fatty acids, sugars, sugar alcohol, organic acids, cholesterol, glycerol and urea.

Sample preparation for GC-MS : The sample preparation for GC-MS analysis was performed according to previous studies with few modifications (Gao et al., 2014). Serum was thawed at $4^{\circ} \mathrm{C}$ and mixed well before use. About $100 \mu \mathrm{l}$ of serum was subjected to protein precipitation using $250 \mu$ of acetonitrile and centrifuged at $12,000 \mathrm{~g}$ for 10 minutes at $4^{\circ} \mathrm{C}$. Then, a volume of $200 \mu$ supernatant was transferred to a tube and evaporated to dryness under the stream of nitrogen prior to derivatization. All the dried samples were derivatized with $30 \mu \mathrm{l}$ methoxylamine hydrochloride $\left(20 \mathrm{mg} / \mathrm{ml}\right.$ in pyridine) at $70^{\circ} \mathrm{C}$ for 60 minutes, followed by $50 \mu \mathrm{l}$ MSTFA (N-Methyl-N (trimethylsilyl) trifluoracetamide) at $40{ }^{\circ} \mathrm{C}$ for 90 minutes. After derivatization samples were cooled at room temperature and $1 \mu \mathrm{l}$ was injected into the GC-MS.

The samples were analysed using a Shimadzu GC-2010 Plus gas chromatography instrument coupled to a Shimadzu QP2010 mass spectrometer (Shimadzu, Japan). The column used for analyses was a DB-5MS fused - silica capillary column ( $30 \mathrm{~m} \times 0.25 \mathrm{~mm}$ I.D, $0.25 \mu \mathrm{M}$ film thicknesses) chemically bonded with a $5 \%$ phenyl $95 \%$ methylpolysiloxane cross-linked stationary phase. Helium was used as the carrier gas with a flow rate of 1.0 $\mathrm{ml} \mathrm{min}-1$. The initial temperature was held at $100^{\circ} \mathrm{C}$ for $4 \mathrm{~min}$, increased to $270^{\circ} \mathrm{C}$ at the rate of $5^{\circ} \mathrm{C} /$ minute for 15 minutes. The temperatures of injection, interface and ion source were set at $280^{\circ} \mathrm{C}, 250^{\circ} \mathrm{C}$ and $200^{\circ} \mathrm{C}$ respectively. One microlitre of the sample was injected in the 10:1 split mode, and the solvent delay time was set to $9 \mathrm{~min}$. The mass spectrometer was operated in electron ionisation mode (electron energy of $70 \mathrm{eV}$ ) and was implemented with the range of $\mathrm{m} / \mathrm{z}$ of 35 to 800 . The identification of the metabolites was based on NIST \& WILEY mass library.

Statistical analysis : Quantitative data were expressed as mean $\pm \mathrm{SD}$ (standard deviation). The levels of significance between the groups were determined by performing ANOVA (Analysis of Variance) and subsequent post hoc (Scheffe's test) analysis using SPSS (version 16.0).

\section{Results and Discussion}

Forced swim test is an established test for depressive animal model. The longer the immobility time, more the depression. The immobility (floating) time was measured as an estimate of phenotypes of depression. Zhoa et al., (2015) reported higher immobility time in case of depressed rats and
Table 1 : Effect of exercise on behavioural changes in depression induced animal models

\begin{tabular}{|c|c|c|}
\hline & FST & SCT \\
\hline Groups & $\begin{array}{l}\text { Duration of mobility } \\
\text { in seconds }\end{array}$ & $\begin{array}{l}\text { Sucrose } \\
\text { preference in \% }\end{array}$ \\
\hline Normal control & $47.96 \pm 1.76$ & $92.04 \pm 1.45$ \\
\hline $\begin{array}{l}\text { Depression induced } \\
\text { Depression + }\end{array}$ & $114.06 \pm 8.22^{* *}$ & $66.91 \pm 0.67^{* *}$ \\
\hline exercise treated & $76.69 \pm 4.20^{* *}$ & $82.10 \pm 0.82^{* *}$ \\
\hline
\end{tabular}

FST - Forced Swim Test; SCT - Sucrose Consumption Test. Data are expressed in mean $\pm S D(n=6),{ }^{* *} p<0.01-$ significant

lower levels in antidepressant (imipramine) - treated rats. In the present study, in FST, significant $(p<0.05, p<0.01)$ increase (114.06 \pm 8.22 seconds) was found in the immobility time of group II (depressed) rats which after subjecting to swimming regimen for four weeks showed a significant reduction (76.69 \pm 4.20 seconds). This indicates that exercise ameliorates depression like behaviour induced by chronic stress (Table 1).

In Sucrose Consumption Test, stressed animal models of depression showed less preference to sucrose consumption 'anhedonia' which was reversed by antidepressant treatment (Willner 1997). In a previous study decreased sucrose consumption found in CUMS induced rats reverted after the swimming exercise which alleviated depression-like behaviour (Lie et al., 2013). Likewise in the present study also, the preference for sucrose consumption was found to decrease $(66.91 \pm 0.67 \%)$ significantly $(p<0.05)$ in group II rats when compared to control group (group I). After 4 weeks of exercise regimen sucrose consumption had increased significantly (82.10 $\pm 0.82 \%$ ) in group III stressed rats, showing that the exercise reduces the Chronic Unpredictable Mild Stress induced depression - like behaviour.

Having seen that exercise produced improvements in the above two behavioural tests, serum metabolic profiling of control, CUMS and exercise groups were carried out by GC-MS analysis. Twenty nine metabolites were identified and they were subjected to one way ANOVA followed by post hoc (Scheffe's test) analysis, which revealed differences in 20 serum metabolites of 3 groups viz., normal, depressed and exercised rats in aminoacids, fatty acids, sugars, sugar alcohol and organic acid metabolite levels (Table 2).

Aminoacids play an important role in human metabolome as they are the precursors of brain neurotransmitters and hence, have been studied as biomarkers for several disorders including schizophrenia (Do et al., 1995), Major depressive disorder Altamura et al., 1995; Xu et al. (2012), Alzhemier's disease Fonteh et al. (2007), cancer Leichtle et al. (2012) and metabolic syndrome Cheng et al. (2012). In a recent study (Batch et al., 2014) reported the significance of Branched chain aminoacids (BCAs) as specific biomarker for certain diseases and observed decreased levels of BCAs and reduced activation of Mammalian 
Table 2 : Serum metabolites detected by GCMS in the depressed and exercised rats

\begin{tabular}{|c|c|c|c|c|c|}
\hline \multirow[b]{2}{*}{ Compounds } & \multirow[b]{2}{*}{ Control $^{*}$} & \multicolumn{2}{|c|}{ Concentration $\left(\mathrm{mmol} \mathrm{ml}^{-1}\right)$} & \multirow[b]{2}{*}{$P$ value } & \multirow[b]{2}{*}{$\%$ change after exercise } \\
\hline & & Depression ${ }^{*}$ & Exercise $^{*}$ & & \\
\hline \multicolumn{6}{|l|}{ Aminoacids } \\
\hline Alanine & $1.49 \pm 0.15$ & $1.32 \pm 0.157$ & $1.43 \pm 0.105$ & $0.378^{\text {ns }}$ & 7.69 \\
\hline Glycine & $0.12 \pm 0.03$ & $0.47 \pm 0.09^{* *}$ & $0.19 \pm 0.06$ & $0.001^{* *}$ & -147.37 \\
\hline Isoleucine & $0.93 \pm 0.07$ & $0.75 \pm 0.08$ & $0.77 \pm 0.8$ & $0.062^{\mathrm{ns}}$ & 2.59 \\
\hline Leucine & $2.44 \pm 0.18$ & $1.38 \pm 0.15$ & $1.70 \pm 0.04$ & $0.000^{* *}$ & 18.82 \\
\hline Proline & $1.01 \pm 0.10$ & $0.073 \pm 0.02^{* *}$ & $0.92 \pm 0.09$ & $0.000^{* *}$ & 92.07 \\
\hline Serine & $0.39 \pm 0.18$ & $5.01 \pm 0.16^{* *}$ & $0.94 \pm 0.02^{\star *}$ & $0.000^{* *}$ & -432.98 \\
\hline Threonine & $0.21 \pm 0.105$ & $0.97 \pm 0.18$ & $0.32 \pm 0.14$ & $0.020^{*}$ & -203.13 \\
\hline Valine & $1.53 \pm 0.11$ & $0.66 \pm 0.05$ & $1.03 \pm 0.40$ & $0.000^{* *}$ & 35.92 \\
\hline \multicolumn{6}{|l|}{ Fatty Acids } \\
\hline Arachidonic acid & $1.43 \pm 0.15$ & $1.073 \pm 0.01^{* *}$ & $1.479 \pm 0.19^{\mathrm{ns}}$ & $0.060^{\mathrm{ns}}$ & 27.45 \\
\hline Linoleic acid & $0.25 \pm 0 . .4$ & $0.18 \pm 0.026^{*}$ & $0.40 \pm 0.032$ & $0.001^{* *}$ & 55.00 \\
\hline Oleic acid & $0.26 \pm 0.04$ & $0.08 \pm 0.13^{* *}$ & $0.23 \pm 0.07$ & $0.003^{* *}$ & 65.22 \\
\hline Palmitic acid & $4.13 \pm 0.14$ & $3.16 \pm 0.25^{\star}$ & $3.77 \pm 0.16$ & $0.044^{*}$ & 16.18 \\
\hline Stearic acid & $1.21 \pm 0.05$ & $1.02 \pm 0.07$ & $1.20 \pm 0.08$ & $0.027^{*}$ & 15.00 \\
\hline Tetradecanoic acid & $0.04 \pm 0.22$ & $0.06 \pm 0.01$ & $0.07 \pm 0.10$ & $0.722^{\text {ns }}$ & 14.28 \\
\hline \multicolumn{6}{|l|}{ Sugars } \\
\hline Fructose & $1.27 \pm 0.2$ & $0.85 \pm 0.04^{* *}$ & $1.13 \pm 0.115$ & $0.020^{* *}$ & 24.78 \\
\hline Galactose & $13.97 \pm 0.11$ & $7.35 \pm 0.24$ & $11.23 \pm 0.15$ & $0.000^{* *}$ & 34.55 \\
\hline Glucose & $12.6 \pm 0.45$ & $8.53 \pm 0.115^{\star *}$ & $9.72 \pm 0.16^{\star *}$ & $0.000^{* *}$ & 12.24 \\
\hline Mannose & $0.186 \pm 0.02$ & $0.09 \pm 0.01$ & $0.34 \pm 0.04$ & $0.000^{* *}$ & 73.53 \\
\hline Xylitol & $1.44 \pm 0.23$ & $0.36 \pm 0.19$ & $0.93 \pm 0.71$ & $0.010^{*}$ & 61.29 \\
\hline \multicolumn{6}{|l|}{ Sugar Alcohol } \\
\hline Myo-inositol & $3.38 \pm 0.08$ & $0.70 \pm 0.10^{* *}$ & $2.32 \pm 0.03^{* *}$ & $0.000^{* *}$ & 69.83 \\
\hline \multicolumn{6}{|l|}{ Organic Acids } \\
\hline 3-hydroxy butyric acid & $3.64 \pm 0.04$ & $1.66 \pm 0.03^{* *}$ & $2.26 \pm 0.06^{\star *}$ & $0.000^{* *}$ & 26.54 \\
\hline Lactic acid & $12.51 \pm 0.21$ & $7.63 \pm 0.22^{* *}$ & $12.14 \pm 0.19$ & $0.000^{* *}$ & 37.15 \\
\hline Malic acid & $0.09 \pm 0.02$ & $0.08 \pm 0.05$ & $0.07 \pm 0.05$ & $0.932^{\text {ns }}$ & -14.28 \\
\hline Oxalic acid & $0.91 \pm 0.02$ & $1.23 \pm 0.107^{* *}$ & $1.06 \pm 0.15$ & $0.004^{* *}$ & -16.04 \\
\hline Propanoic acid & $0.56 \pm 019$ & $0.42 \pm 0.14$ & $0.75 \pm 0.04$ & $0.069^{\mathrm{ns}}$ & 44.00 \\
\hline Succinic acid & $0.05 \pm 0.03$ & $0.71 \pm 0.06$ & $0.29 \pm 0.08$ & $0.000^{* *}$ & -144.83 \\
\hline \multicolumn{6}{|l|}{ Others } \\
\hline Cholesterol & $1.33 \pm 0.16$ & $1.22 \pm 0.05$ & $1.25 \pm 0.04$ & $0.060^{\mathrm{ns}}$ & 2.40 \\
\hline Glycerol & $1.8 \pm 0.19$ & $1.52 \pm 0.16$ & $1.35 \pm 0.20$ & $0.064^{\mathrm{ns}}$ & -12.59 \\
\hline Urea & $11.20 \pm 0.16$ & $11.72 \pm 0.11$ & $12.09 \pm 0.70$ & $0.104^{\mathrm{ns}}$ & 3.06 \\
\hline
\end{tabular}

$\uparrow, \downarrow$ indicate significant increase and decrease in depressed rats when compared to healthy rats. Data are expressed as mean $\pm \operatorname{SD}(n=6)$,

${ }^{*} p<0.05,{ }^{* *} p<0.01$ - significant, ${ }^{\text {ns }}-$ not significant, ${ }^{*} n=6$ for each group

target of rapamycin (mTor) ubiquitous serine/threonine protein kinase. This shows that BCAs play a crucial unrecognized role in the etiology of depression (Baranyi et al., 2016). Likewise in the present study, BCAs such as leucine $\left(1.38 \mathrm{mmolm}^{-1}\right)$ and valine $(0.66$ $\mathrm{mmolml}^{-1}$ ) were found to be decreased in stress induced group. After the exercise, the BCAs level was found to be increased significantly, which may due to the increased activation of $m$ Tor.

Excitatory neurotransmitters such as serine (5.01 $\left.\mathrm{mmolml}^{-1}\right)$ and glycine $\left(0.47 \mathrm{mmolml}^{-1}\right)$ are found to be higher in depressed animals when compared to control. Serine and glycine, one of the excitatory amino acids in brain are implicated in neurotransmission acting either as neurotransmitters or as modulators of NMDA receptors for excitation. Previous studies have reported increase levels of excitatory amino acids such as serine and glycine in major depressive disorder (Mauri et al., 1998; Hashimoto et al., 2016) and after the exercise regimen, there was a significant decrease in their levels when compared to that of the depressed rats. Exercise, as found in this study, had brought down the increased levels of these excitatory aminoacids (serine and glycine) almost to normal levels in depressed rats.

Stearic acid seems to possess neuroprotective effects (Wang et al., 2006). In this study, decreased concentrations of 
fatty acids such as palmitic acid $\left(3.16 \mathrm{mmol} \mathrm{ml}^{-1}\right)$, stearic acid $\left(1.02 \mathrm{mmol} \mathrm{ml}^{-1}\right)$, linoleic acid $\left(0.18 \mathrm{mmol} \mathrm{ml}^{-1}\right)$ and oleic acid $(0.08$ $\mathrm{mmolml}^{-1}$ ) was observed in depressed rats when compared to control rats. These results suggest of alterations in fatty acid metabolism in depressed rats as they are associated with metabolic pathways related to TCA cycle (Serretti et al., 2004). Previous metabolic studies have reported that fatty acid metabolism is significantly lower and shifted from $\beta$ - oxidation to $\omega$-oxidation in depressed patients, in comparison to nondepressed controls (Maes et al., 1996; Paige et al.,2007; Steffens et al., 2010). Thus, decreased serum fatty acids may reduced result in oxidation of fatty acids, which in turn reduced the production of acetyl CoA that leads to dysfunction of TCA cycle, a possible cause for fatigue, which is most frequently representated symptom in major depressive disorder (Serretti et al., 2004). After 28 days of swimming, the fatty acid levels were found to increase significantly which in turn resulted in increased $\beta$ - oxidation leading to increased TCA cycle pathway with subsequent relief from fatique.

Myo-inositol, a key metabolic precursor to phosphoinositide pathway was reported to be decreased in major depression (Coupland et al., 2005). Decreased levels of myoInositol have been reported in CSF and post-mortem frontal cortex of patients with mood disorders (Barkai et al., 1978; Shimon et al., 1997). Glial cells contain a high concentration of myo-Inositol (Brand et al., 1993; Griffin et al., 2002) and it contributes to glial osmoregulatory functioning (Strange, 1992; Fisher et al., 2002), as well as a wide range of other structural and signalling functions. Stress induced disruption of glial-neuronal interaction lead to glial deficits in mood regulation (Edgar and Sibille, 2012), may be due to reduced myo-inositol levels in the brain. this in turn might result in reduced osmoregulatory and cell signalling functions of myoinositol. Accordingly, in the present study decreased level $(0.70$ $\mathrm{mmolm}^{-1}$ ) of myo-inositol was found in depressed rats possibly due to glial dysfunction and the same improved $\left(2.32 \mathrm{mmolml}^{-1}\right)$ significantly after exercise regimen, with concurrent improvement in depressive behaviour(Table2).

A previous study reported decreased levels of plasma glucose and lactate in major depressive disorder that results in putative imbalance in glycolysis and gluconeogenesis (Zheng et al., 2012). Likewise, in this study, levels of other metabolites viz., sugars such as glucose $\left(8.53 \mathrm{mmolml}^{-1}\right)$, fructose $\left(0.85 \mathrm{mmolml}^{-1}\right)$, Galactose $\left(7.35 \mathrm{mmolml}^{-1}\right)$, Mannose $\left(0.09 \mathrm{mmolm}^{-1}\right)$, Xylitol $\left(0.36 \mathrm{mmolml}^{-1}\right)$ and the organic acids lactate $\left(7.63 \mathrm{mmolm}^{-1}\right)$, 3-hydroxy butyric acid $\left(1.66 \mathrm{mmolml}^{-1}\right)$ decreased significantly in depressed rats and their levels increased after swimming. Neverthless, in the present study no significant levels in the baseline levels of metabolites (glycerol, malic acid, propanoic acid, arachidonic acid, Cholesterol, Tetradecanoic acid, alanine, isoleucine and urea) was found among the healthy control, depressed and exercised groups of rats.

GCMS metabolomic analysis carried out in this study found significant differences in few unique metabolites among aminoacids, sugars, fatty acids, sugar alcohols and organic acids between the groups viz normal control, depressed and exercised rats. Identifying the biochemical mechanisms of these both in depression and after exercised would enhance our understanding of the etiology of depression and if validated, may become useful diagnostic bio-markers for improving the treatment of depression, as well. This presents new opportunities for translational research in depression and schizophrenia.

\section{Acknowledgements}

We acknowledge the financial support given by University Grant Commission (UGC), New Delhi, for providing financial support in the form of minor research project No. F.NO:44/2013-14(MRP-SEM/UGC-SERO) and also thank Central Research Laboratory, PSG College of Arts \& Science, Coimbatore for GC-MS facility.

\section{References}

Altamura, C., M. Maes., J. Dai and H.Y. Meltzer: Plasma concentrations of excitatory amino acids, serine, glycine, taurine and histidine in major depression. Eur. Neuropsychopharmacol., 5, 71 - 75 (1995).

Anisman, H. and K. Matheson: Stress, depression, and anhedonia: Caveats concerning animal models Neurosci. Biobehav. Revs., 29, 525-546 (2005).

Baranyi, A., O.A. Ghadikolai, D. Von Lewinski, H.B. Rothenhausler, S.Theokas, C. Robier, H. Mangge, G. Reicht, P. Hlade and A. Meinitzer : Branached - chain animoacids as new biomarkers of major depression-Anovel neurobiology of mood disorders. PLOS ONE, 11, e0160542 (2016).

Barkai, A.I., D.L. Dunner, H.A. Gross, P. Mayo and R.R. Fieve : Reduced myo-inositol levels in cerebrospinal fluid from patients with affective disorder. Biol. Psychiatry., 13, 65-72 (1978).

Batch, B.C., K. Hyland and L.P. Svetkey: Branch chain aminoacids: Biomarkers of health and disease. Curr. Opin. Clin. Nutr. Metab. Care., 17, 86-89 (2014).

Brand, A., C. Richter-Landsberg and D. Leibfritz : Multinuclear NMR studies on the energy metabolism of glial and neuronal cells. Dev. Neurosci., 15, 289-298(1993).

Cheng, S., E.P. Rhee, M.G. Larson, G.D. Lewis, E.L. McCabe, D. Shen, M.J. Palma, L.D. Roberts, A. Dejam, A.L. Souza, A.A. Deik, M. Magnusson, C.S. Fox, C.J. O'Donnell, R.S. Vasan, O. Melander, C.B. Clish, R.E. Gerszten and T.J. Wang : Metabolite profiling indentifies pathways associated with metabolic risk in humans. Circulation, 125, 2222-2231 (2012).

Coles, M.E. and R.G. Heimberg : Memory biases in the anxiety disorders: current status. Clin. Psychol. Rev., 22, 587-627 (2002).

Contarteze, R.V., B. Manchado Fde, C.A. Gobatto and M.A. De Mello : Stress biomarkers in rats submitted to swimming and treadmill running exercises. Compar. Bioche. Physio., Part A : Molec. Integ. Physio., 151, 415-22 (2008).

Cotman, C.W., N.C. Berchtold and L.A. Christie: Exercise builds brain health : Key roles of growth factor cascades and inflammation. Trends Neurosciences, 30, 464-472 (2007).

Coupland, N.J., C.J. Ogilvie, K.M. Hegadoren, P. Seres, C.C. Hanstock and P.S. Allen : Decreased prefrontal myoinositol in major depressive disorder. Biol. Psychiatry., 57, 1526-1534 (2005).

Do, K.Q., C.J. Lauer, W. Schreiber, M. Zollinger, U. Gutteck-Amsler, M. Cuenod and F. Holsbeer : gamma-Glutamylglutamine and taurine concentrations are decreased in the cerebrospinal fluid of drug- 
naive patients with schizophrenic disorders. J. Neurochem., 65 , 2652-2662 (1995).

Edgar, N. and E. Sibille: A putative functional role for oligodendrocytes in mood regulation. Transl. Psychiatry, 2, 109 (2012).

Eyre, H. and B.T. Baune: Neuroimmunological effects of physical exercise in depression. Brain Behav. Imm., 26, 251 - 266 (2012).

Fisher, S.K., J.E. Novak and B.W. Agranoff: Inositol and higher inositol phosphates in neural tissues: Homeostasis, metabolism and functional significance. J. Neurochem., 82, 736-754 (2002).

Fonteh, A.N., R.J. Harrington, A. Tsai, P. Liao and M.G. Harrington: Free amino acid and dipeptide changes in the body fluids from Alzheimer's disease subjects. Amino Acids, 32, 213-224 (2007).

Gao, X., B. Guo, L. Yang, J. Liu, X. Zhang, X. Qin and G. Du : Selection and dynamic metabolic response of rat biomarkers by metabonomics and multivariate statistical analysis combined with GC-MS. Pharmac. Bioche. Behav., 117, 85-91 (2014).

Griffin, J.L., M. Bollard, J.K. Nicholson and K. Bhakoo : Spectral profiles of cultured neuronal and glial cells derived from HRMAS (1) $\mathrm{H}$ NMR spectroscopy. NMR Biomed., 15, 375-384 (2002) .

Hashimoto, K., T. Yoshida, M. Ishikawa, Y. Fugita, T. Niitsu, M. Nakazato, H.Watanabe, T. Sasaki, A. Shiina, T. Hashimoto, N. Kanahara, T. Hasegawa, M. Enohara, A. Kimura and M. Iyo : Increased serum levels of serine enantiomers in patients with depression. Acta Neuropsychiatr., 28, 173-78 (2016)

Kaddurah-Daouk, R., B.S. Kristal and R.M. Weinshilboum: Metabolomics: A global biochemical approach to drug response and disease. Annu. Rev. Pharmacol. Toxicol., 48, 653-683 (2008).

Kell, D.B.: Systems biology, metabolic modelling and metabolomics in drug discovery and development. Drug Discove. Today, 11, 1085$1092(2006)$

Leichtle, A.B., J.M. Nuoffer, U. Cegalarek, J. Kase, T. Conrad, H. Witzigmann, J. Thiery and G.M. Fiedler: Serum amino acid profiles and their alterations in colorectal cancer. Metabolomics, 8, 643653 (2012).

Liu, X., J. Yang le, S.J. Fan, H. Jiang and F. Pan : Swimming exercise effects on the expression of HSP70 and iNOS in hippocampus and prefrontal cortex in combined stress. Neuroscience, 476, 99-103 (2010).

Liu, W., H. Sheng, Y. Xu, Y. Liu, J. Lu and X. Ni : Swimming exercise ameliorates depression-like behaviour in chronically stressed rats: Relevant to proinflammatory cytokines and IDO activation. Behavi. Brain Res., 242, 110-116 (2013).

Maes, M., R. Smith, A. Christophe, P. Cosyns, R. Desnyder and H.Y. Meltzer: Fatty acid composition major depression: Decreased $\omega 3$ fractions in cholesteryl esters and increased c20:4 $\omega 6 / c 20: 5 \omega 3$ ratio in cholesteryl esters and phospholipids. J. Affect Disord., $\mathbf{3 8 ,}$ 35-46 (1996).

Mauri, M.C., A. Ferrara, L. Boscati, S. Bravin, F. Zamberlan, M. Alecci and G. Invernizzi : Plasma and platelet amino acid concentrations in patients affected by major depression and under fluvoxamine treatment. Neuropsychobiology, 37, 124-129 (1998).

Paige, L.A., M.W. Mitchell, K.R. Krishnan, R. Kaddurah-Daouk and D.C. Steffens: A preliminary metabolomics analysis of older adults with and without depression. Int. J. Geriatr. Psychiatry., 22, 418-423 (2007).

Pasikanti, K.K., P.C. Ho and E.C. Chan : Gas chromatography/ mass spectrometry in metabolic profiling of biological fluids. J.
Chromatogr. B. Analyt. Technol. Biomed. Life Sci., 871, 202 - 211 (2008).

Porsolt, R.D., A. Bertin and M. Jalfre: Behavioral despair in mice: A primary screening test for antidepressants. Arch. Int. Pharmacodyn. Ther., 229, 327-36 (1977).

Redrobe, J.P., M. Bourin., M.C. Colombel and G.B. Baker : Dosedependent noradrenergic and serotonergic properties of venlafaxine in animal models indicative of antidepressant activity. Psychopharmacology (Berl), 138, 1-8 (1998).

Reynolds, E.H. : Brain and mind : A challenge for WHO. Lancet., 361, 1924-5(2003).

Schwartz, E. and S. Bahn: The utility of biomarker discovery approaches for the detection of disease mechanisms in psychiatric disorders. Br. J. Pharmacol., 153, S133-S136 (2008).

Serretti, A., L. Mandelli., E. Lattuada and E. Smeraldi: Depressive syndrome in major psychoses: A study on 1351 subjects. Psychiatry Res., 127, 85-99 (2004).

Shenl, B.V., D.W. Harrison and H.D. Demaree: The neuropsychology of depression: a literature review and preliminary model. Neuropsychol., 13, 33-42 (2003).

Shimon, H., G. Agam, R.H. Belmaker, T.M. Hyde and J.E. Kleinman : Reduced frontal cortex inositol levels in postmortem brain of suicide victims and patients with bipolar disorder. Am. J. Psychiatry, 154, 1148-1150 (1997).

Steffens, D.C., W. Jiang, K.R.R. Krishnan, E.D. Karoly, M.W. Mitchell, C.M. Connor and R. Kaddurah-Daouk: Metabolomic differences in heart failure patients with and without major depression. J. Geriatr. Psychiatry Neurol., 23, 138-146 (2010).

Strange, K.: Regulation of solute and water balance and cell volume in the central nervous system. J. Am. Soc. Nephrol., 3, 12-27 (1992).

Wang, Z.J., G.M. Li, W.L. Tang and M. Yin : Neuroprotective effects of stearic acid against toxicity of oxygen/glucose deprivation or glutamate on rat cortical or hippocampal slices. Acta. Pharmacol. Sin., 27, 145-150 (2006).

Willner, P. : Validity, reliability and utility of the chronic mild stress model of depression : A 10-year review and evaluation. Psychopharmacology (Berl), 134, 319-329 (1997).

Willner, P., A. Towell, D. Sampson, S. Sophokleous and R. Muscat: Reduction of sucrose preference by chronic unpredictable mild stress and its restoration by a tricyclic antidepressant. Psychopharmacology, 93, 358-64 (1987).

Wingenfeld, K. and O.T. Wolf: HPA axis alterations in mental disorders: impact on memory and its relevance for therapeutic interventions. CNS Neurosci. Ther., 17, 714-722 (2011).

Xu, H.B., L. Fang, Z.C. Hu, Y.C. Chen, J.J. Chen, F.F. Li, J. Lu, J. Mu and P. Xie : Potential clinical utility of plasma amino acid profiling in the detection of major depressive disorder. Phychiatry Res., 200, 1054-1057 (2012)

Zheng, P., H.C. Gao, Q. Li, W.H. Shao, M.L. Zhang, K. Cheng, Y. Yang, S.H. Fan, L. Chen, L. Fang and P. Xie : Plasma metabonomics as a novel diagnostic approach for major depressive disorder. J. Proteome. Res.,11, 1741-1748 (2012).

Zhao, J., Y. Jungi, C. Jangi, K. Chun, S. W. Kwon and J. Lee : Metabolomic identification of biochmemical changes induced by fluoxetine and imipramine in a chronic mild stress mouse model of depression. Sci. Rep., 5, 8890 (2015). 